\title{
Role of Dexmedetomidine in anesthetic management of lower limb orthopaedic surgery in a patient with low ejection fraction
}

\author{
Kriti Nagar $^{1, *}$, Shirin Parveen ${ }^{2}$ \\ ${ }^{\mathbf{1}}$ Junior Resident, ${ }^{\mathbf{2}}$ Assistant Professor, Dept. of Anaesthesiology and Critical Care, Era's Lucknow Medical College and Hospital,
} Lucknow, Uttar Pradesh, India

*Corresponding Author:

Email: kriti.1188@gmail.com

Received: $13^{\text {th }}$ September, 2017

Accepted: $05^{\text {th }}$ December, 2017

\begin{abstract}
Cardiac patient for non cardiac surgery has since forever been a topic of interest for the anaesthesiologist. Few cases have only been reported with their own suitable line of management, however the simultaneous use of combined epidural spinal anaesthesia and Dexmedetomidine, an $\alpha 2$ agonist has been studied by only a handful. Use of epidural for incremental doses of local anaesthetic for allaying the postoperative pain and thereby reducing the occurrence of ischemic events is also being described.
\end{abstract}

Keywords: Ischaemic Heart Disease, Epidural Anaesthesia, Ejection Fraction, Dexmedetomidine.

\section{Introduction}

Ischemic Heart Disease (IHD) is a leading cause of perioperative morbidity and mortality in patients coming for non cardiac surgery ${ }^{1}$ Augmented hemodynamic control with intensive perioperative pharmacologic therapy with beta-blockers and possibly alpha-2 agonist has been shown to improve perioperative cardiovascular outcomes. ${ }^{2}$ Management of such patients requires thorough clinical evaluation, medical therapy, identification of risk factors, monitoring and appropriate mode of anaesthesia. There have been very few cases reported of combined Spinal Epidural Anaesthesia for patients with IHD undergoing non cardiac surgery. Here we report a successful anesthetic management of a patient with a history of MI and ejection fraction of $40 \%$ posted for CRIF with nailing of Tibia under combined spinal epidural anesthesia and iv Dexmedetomidine.

\section{Case Report}

A 55 year old male patient with fracture Tibia and Fibula of left lower limb was posted for CRIF with nailing. The patient was a known case of IHD with a history of MI (inferior wall MI, posterior wall MI, ST elevation in leads II, III, aVF) 6 years back. Coronary interevention was not done and he was started on Tab. Metoprolol $50 \mathrm{mg}$ OD, Tab Aspirin 75mg OD and Tab Atorvastatin $20 \mathrm{mg}$ OD. On examination, Heart Rate was $82 \mathrm{bpm}$, BP 140/90 mmHg, Oedema absent, Mallampati Grade III, partially edentulous, airway adequate and Spine was clinically normal. Laboratory investigations(complete blood counts, liver function tests, renal function tests, coagulation profile and blood sugar) were within normal limit, chest $\mathrm{x}$ ray showed no abnormality and ECG showed normal sinus rhythm with heart rate of $68 \mathrm{bpm}$, abnormal Q waves (II, aVF) and inverted $\mathrm{T}$ waves(V6). A cardiology reference was sought thereafter and 2D Echo was done which revealed poor echo window, normal valves, dilated left ventricle, right wall motion abnormality present (inferior wall hypokinesia), Ejection fraction $40 \%$, LVDD - I. Further Coronary Angiography revealed OSTIA c LAD- $50 \%$, DL- $80 \%$, OM-100\%, RCA-70\%. Case was accepted to be taken with $>5 \%$ risk of cardiac risk index under ASA grade III.

\section{Anesthetic Management}

Prior to the surgery, the risk associated with the surgery was explained and written and informed consent was obtained. Patient was instructed to take Tab Alprazolam $0.5 \mathrm{mg}$ HS night before surgery and to continue Tab Metoprolol and Tab Aspirin on the day of surgery. The patient was then shifted to pre operative ward where his baseline hemodynamic parameters were noted, Heart Rate of $72 \mathrm{bpm}$, Blood Pressure 140/90 $\mathrm{mmHg}$, and oxygen saturation of $98 \%$ on room air. Intravenous access was secured using $18 \mathrm{G}$ cannula and Ringer Lactate was stared at $2 \mathrm{ml} / \mathrm{kg} / \mathrm{hr}$. Thereafter, the patient was shifted to the Operation Theatre where all cardiac emergency drugs, defibrillator and equipments for resuscitation were kept ready. Electrocardiography, Heart Rate, Non Invasive Blood Pressure and $\mathrm{SpO} 2$ monitoring was done. Procedure was explained to the patient. Under all aseptic precautions with the patient in sitting position, $1.5 \mathrm{ml}$ of $2 \%$ lignocaine infiltrated in L2-L3 interspace. Epidural space entered with BBraun 18G Tuohy's epidural needle with Loss of Resistance technique and $20 \mathrm{G}$ epidural catheter was passed upto $4 \mathrm{~cm}$ mark through the needle. The catheter was secured in place at $10 \mathrm{~cm}$ mark following negative aspiration for blood and cerebrospinal fluid. A 25G spinal needle introduced in L3-L4 interspace. After free flow of CSF, $0.5 \%$ inj. Bupivacaine Heavy $1.2 \mathrm{ml}$ along with inj. Fentanyl $25 \mu \mathrm{g}$ was given intrathecally. The patient was 
moved to supine position and oxygen was administered via Hudson mask. Subarachnoid block was achieved till T8 level. The usual side effects like hypotension and tachycardia were not noted with low dose spinal anaesthesia. Inj. Midazolam 1mg and Inj Fentanyl 25 $\mu \mathrm{g}$ were given intravenous. After patient was hemodynamically stable, Dexmedetomidine $(1 \mu \mathrm{g} / \mathrm{kg})$ was given intravenous over 10 minutes and thereafter an infusion was started at $0.4 \mu \mathrm{g} / \mathrm{kg} / \mathrm{hr}$. Heart Rate was maintained between 70-80 bpm, Systolic Blood Pressure between 100-110 mmHg and Diastolic Blood Pressure between 66-74 mmHg. After an hour of administration of spinal anaesthesia, $0.125 \%$ Bupivacaine $7.5 \mathrm{mg}$ given via epidural. The surgery lasted for about 90 minutes. With the completion of surgery, Dexmedetomidine infusion was stopped and the patient was shifted to post operative ward. In the post operative period, $0.125 \%$ Bupivacaine $7.5 \mathrm{mg}$ was given as intermittent bolus every 2 hourly upto 8 hours and 3 hourly thereafter for next 9 hours for pain relief. Epidural catheter was removed after 24 hours. Post operative period was uneventful.

\section{Discussion}

The goals for anesthesia for a patient with IHD are to maintain stable hemodynamics, optimize myocardial oxygen supply and reduce oxygen demand, monitor for ischemia, treat ischemia or infarction if it develops, and maintain normothermia. Selection of drugs should be with the objective of minimizing demand, and optimum supply of oxygen. ${ }^{1}$

Most of the intravenous anaesthetic agents have a direct depressant action on the myocardium and may also reduce vascular tone. This results in hypotension (especially in the hypovolaemic patient), often with a compensatory tachycardia, which may cause myocardial ischaemia. ${ }^{3}$

The use of regional anaesthesia has its own advantages like epidural anaesthesia which reduces preload and afterload, coagulation responses, and in the case of thoracic epidurals, causes coronary vasodilation. These effects should reduce perioperative myocardial ischaemia, but this is not supported by research. However, good epidural analgesia may reduce the incidence of tachycardias arising due to postoperative pain. ${ }^{3}$

Increased attention should be focused on the postoperative period to reduce perioperative cardiac morbidity. ${ }^{4}$ Perioperative $\mathrm{MI}$ is a significant issue not only in patients undergoing high risk surgery but also in those with minor surgical interventions and it also can be silent; its ECG changes are frequently transient and minor troponin elevations predict early and late morbidity and mortality. ${ }^{5}$

\section{Conclusion}

Regional anaesthesia appears to be safer compared to General Anaesthesia (GA) in a cardiac patient for non cardiac surgery as it maintains the myocardial oxygen supply and reduces the oxygen demand, eliminates the hypotension caused during induction and hypertension and tachycardia due to pressor response caused by laryngoscopy. It also helps in the management of post operative pain thereby reducing the risks of tachycardia, myocardial ischemia and postoperative MI. But due to uncontrolled sympathetic block there may be hypotension, which can be prevented by minimal sympathetic block with appropriate dose of intrathecal bupivacaine combined with epidural for supplemental doses. Intra operative dexmedetomidine infusion decreases heart rate and blood pressure that may be advantageous in ischemic heart disease by improving oxygen supply and demand balance.

\section{References}

1. Kaul TK, Tayal G. Anaesthetic considerations in cardiac patients undergoing non cardiac surgery. Indian J Anaesth 2007;51:280-6.

2. Akhtar S, Silverman DG. Crit Care Med. 2004 Apr;32(4 Suppl):S126-36.

3. Koh SH, Rogers J. Anaesthesia for patients with cardiac disease undergoing non cardiac surgery. Update in Anaesthe

4. Mangano DT, Browner WS, Hollenberg M, London MJ, Tubau JF, Tateo IM. Association of peripoerative myocardial ischaemia with cardiac morbidity and mortality in men undergoing noncardiac surgery. The study of perioperative ischemia research group. N Engl J Med 1990;323:1781-8.

5. Landesberg G. The pathophysiology of perioperative myocardial infarction: Facts and perspectives. $\mathrm{J}$ Cardiothorac Vasc Anesth 2003 Feb;17(1):90-100. 